SISSA $/$ AP $/ 95 / 117$

hep-ph/yymmnn

\title{
Towards a Nonequilibrium Quantum Field Theory Approach to Electroweak Baryogenesis
}

\author{
ANTONIO RIOTTOH \\ International School for Advanced Studies, SISSA-ISAS, \\ Strada Costiera 11, I-34014, Miramare, Trieste, Italy \\ and \\ Istituto Nazionale di Fisica Nucleare, Sezione di Padova, 35100 Padova, Italy
}

\begin{abstract}
We propose a general method to compute $C P$-violating observables from extensions of the standard model in the context of electroweak baryogenesis. It is alternative to the one recently developed by Huet and Nelson and relies on a nonequilibrium quantum field theory approach. The method is valid for all shapes and sizes of the bubble wall expanding in the thermal bath during a first-order electroweak phase transition. The quantum physics of $C P$-violation and its suppression coming from the incoherent nature of thermal processes are also made explicit.
\end{abstract}

*riotto@tsmi19.sissa.it. Address after November 95: Theoretical Astrophysics Group, NASA/Fermilab, Batavia, IL60510, USA. 


\section{Introduction}

It is commonly believed that many of the current questions of cosmology can be answered by studying the nontrivial dynamics of the approach to equilibrium in complex systems. Nevertheless, despite their immense relevance, only very recently much effort has been made to understand nonequilibrium phenomena occurred in the early Universe. For instance, recent investigations on the non-linear quantum dynamics of scalar fields have implications for the reheating after the inflationary era and reveal that particle production appears to be significantly different from linear estimates due to the time evolution of the inflation field [1]. The quantum non-linear effects lead to an extremely effective dissipational dynamics and particle production even in the simplest self-interacting theory where the single particle decay is kinematically forbidden.

At the electroweak scale, the focus has been in generation of the baryon asymmetry during a first-order phase transition where the $S U(2)_{L} \otimes U(1)_{Y}$ symmetry is broken down to $U(1)_{\mathrm{em}}$ [2]. Even though there are certain questions related to the reliability of the perturbative expansion for weak enough transitions [3], it is well established that nonequilibrium conditions are a crucial ingredients for baryogenesis.

An interesting attempt to explain the observed baryon to entropy ratio $n_{B} / s \sim 10^{-10}$ was made by Farrar and Shaposhnikov [4] in the context of Standard Model (SM). However, it was later shown [5] that the $C P$-violating processes of quantum interference provided by the Kobayashi-Maskawa matrix were far too slow in comparison with the rapid quark-gluon plasma interactions responsible for the loss of quantistic coherence. In contrast, many extensions of the standard model, like the Minimal Supersymmetric Standard Model, contain additional sources of $C P$ in the Higgs sector (which requires at least two Higgs doublets) and can account for the observed baryon asymmetry.

Both in the case of explicit [6] or spontaneous [7] $C P$-violation, particle mass matrices acquire a nontrivial space-time dependence when bubbles of the broken phase nucleate and expand during a first-order electroweak phase transition. This space-time dependence cannot be rotated away at two adjacent points by the same unitary transformation and gives rise to sufficiently fast nonequilibrium $C P$-violating effects inside the wall of a bubble of broken phase expanding in the plasma. 
Two different limits were originally investigated in the literature. In the case of thin bubble walls, characterized by the condition $\tau / L_{\mathrm{w}} \gg 1$, where $L_{\mathrm{w}}$ and $\tau$ are the wall thickness and the typical particle interaction time, the asymmetric (in fermion numbers) reflection of particles off the bubble wall is the dominant effect [8]. The induced fermion number flux is then reprocessed into a baryon asymmetry by the anomalous $(B+L)$ violating sphaleronic transitions in the unbroken phase [9].

In the opposite limit of thick bubble walls, $\tau / L_{\mathrm{w}} \ll 1$, local operators coupling the baryon current, or related currents, to the space-time derivative of the $C P$-violating phase act as classical $C P$-violating perturbations inside the bubble wall 10,11 and effective chemical potentials account for particle asymmetries.

In both scenarios these $C P$-violating sources are locally induced by the passage of the wall and fuel baryogenesis. They are then inserted into a set of classical Boltzmann equations for particle distribution densities which permit to describe Debye screening of induced gauge charges [12, particle number changing reactions [13] and to trace the crucial role played by diffusion [14]. Indeed, it has been convincingly established that transport allow $C P$-violating charges to efficiently diffuse in front of the advancing bubble wall where anomalous electroweak baryon violating processes are unsuppressed, thus greatly enhancing the final baryon asymmetry.

A new method to compute the effects of $C P$-violation from extensions of SM on the electroweak baryogenesis has been recently developed by Huet and Nelson [15] and subsequently applied to supersymmetric baryogenesis [16]. It takes into account both the effects of scattering from thermal particles and the terms which lead to $C P$-violation in particle propagation, reflecting the interplay between the coherent phenomenon of $C P$ violation and the incoherent nature of plasma physics. It is also valid for generic wall shapes and sizes with a significant improvement over the previous estimates. As a matter of fact, particle interaction times are neither very small nor very large compared to the thickness of the wall and the thin (thick) wall limit can overestimate (underestimate) the amount of baryon asymmetry. Moreover, particles moving with large oblique angles relative to the advancing wall are likely to interact many times when inside the wall and the thin wall limit may not be applied.

When taking the thick wall limit, the method predicts the correct behaviour with the 
mass background in agreement with the results of ref. [11] and evades the assumption that local particle distributions relax towards thermal equilibrium according to some classical equations of motion, which is not well-grounded for particles whose wavelength perpendicular to the wall is larger than their mean free path and makes the classical treatment of $C P$-violation not adequate.

Technically speaking, in refs. 15, 16 $C P$-violating sources were described in terms of quantum mechanical $C P$-violating reflection and transmission from layers of the phase boundary. Thermalization effects on particle distributions are included by averaging over a layer of thickness equal to the free mean path $\Delta=\tau v$ ( $v$ being the particle velocity) the currents $J_{ \pm}$in the rest frame of the wall and resulting from particles moving toward the positive (negative) direction $z$ perpendicular to the wall. For example, the current $J_{+}$receives contributions from either particles originating from the thermal bath at a certain point $z$ an moving with a positive velocity and transmitted to $z+\Delta$ (with the possibility of different scatterings along the path), and from particles originating at $z+\Delta$ and moving with velocity $-v$ and being reflected back towards $z+\Delta$. When boosted to the plasma frame, these currents give rise to the $C P$-violating source terms for the final baryon asymmetry.

It is indisputable that the ultimate answer on electroweak baryogenesis can be provided only by a self-consistent nonequilibrium Quantum Field Theory (QFT) approach. Kinetic theory and classical Boltzmann equations have been used to describe the dynamics of particles treated as classical with a defined position, energy and momentum. However, the validity of the kinetic theory is restricted by the condition that the mean free path of particles must be larger than any other microscopic length scale. In particular, the mean free path must be large compared to the Compton wavelength of the underlying particle in order for the classical picture to be valid, which is not guaranteed for particles with a small momentum perpendicular to the wall. Moreover, since the effects of scattering on particle propagation can be accounted for by substituting particles with quasiparticles with a modified dispersion relation and a given damping rate $\Gamma$ proportional to the imaginary part of the self-energy, only when the energy of quasiparticles is large compared to $\Gamma$ it is possible to speak about coherent excited states and describe them through classical Boltzmann equations opportunely modified to include plasma ef- 
fects. [17]. In the opposite limit, the key elements to address the space-time evolution of distribution functions are provided by the Wigner function techniques which date back to Wigner's work on transport phenomena [18]. Quantum distribution functions are the correct functions to describe particles in an interacting, many-particle environment and obey the quantum Boltzmann equation.

The aim of this paper is to describe a new method to compute the effect of $\mathrm{CP}$ violation coming from extensions of the SM on the mechanism of electroweak baryogenesis and, in these respects, it should be regarded as alternative and complementary to the one proposed in refs. [15, 16]. The method, valid for all wall shapes and sizes, is entirely based on a nonequilibrium QFT diagrammatic approach and naturally incorporates the effects of the incoherent nature of plasma physics on $C P$-violating observables. As such, it should be considered as a first step towards a full nonequilibrium quantum kinetic theory approach to electroweak baryogenesis. For sake of clarity, the method is also illustrated through an example in the framework of the two-Higgs doublet model.

\section{The method}

The ordinary quantum field theory (at finite temperature), which mainly deals with transition amplitudes in particle reactions, is not useful to study the dynamics of classical order parameters. This is because we need their temporal evolution with definite initial conditions and not simply the transition amplitude of particle reactions with fixed initial and final conditions. The most appropriate extension of the field theory to deal with these issues it to generalize the time contour of integration to a closed-time path (CPT). More precisely, the time integration contour is deformed to run from $-\infty$ to $+\infty$ and back to $-\infty$ [19]. The CPT formalism is a powerful Green's function formulation for describing nonequilibrium phenomena in field theory. It allows to describe phase-transition phenomena and to obtain a self-consistent set of quantum Boltzmann equations. The formalism yields various quantum averages of operators evaluated in the in-state without specifying the out-state. As with the Euclidean time formulation, scalar (fermionic) fields $\phi$ are still periodic (anti-periodic) in time, but with $\phi(t, \mathbf{x})=\phi(t-i \beta, \mathbf{x}), \beta=1 / T$. Temperature appears due to boundary condition, but now time is explicitly present in 
the integration contour.

As a consequence of the time contour, we must now identify field variables with arguments on the positive or negative directional branches of the time path. This doubling of field variables leads to four different real-time propagators on the contour [19] for a generic scalar field $\phi$ (and analogously for fermionic fields)

$$
\begin{aligned}
& G_{\phi}^{++}\left(x-x^{\prime}\right)=i\left\langle T_{+} \phi(x) \phi\left(x^{\prime}\right)\right\rangle, \\
& G_{\phi}^{--}\left(x-x^{\prime}\right)=i\left\langle T_{-} \phi(x) \phi\left(x^{\prime}\right)\right\rangle, \\
& G_{\phi}^{+-}\left(x-x^{\prime}\right)=i\left\langle\phi\left(x^{\prime}\right) \phi(x)\right\rangle=G_{\phi}^{>}\left(x-x^{\prime}\right), \\
& G_{\phi}^{-+}\left(x-x^{\prime}\right)=i\left\langle\phi(x) \phi\left(x^{\prime}\right)\right\rangle=G_{\phi}^{<}\left(x-x^{\prime}\right),
\end{aligned}
$$

where $T_{+}$and $T_{-}$indicate the chronological and anti-chronological ordering, $G_{\phi}^{++}$is the usual physical (causal) propagator. The other three propagators come as a consequence of the time contour and are auxiliary (unphysical) propagators.

The explicit expressions for the above propagators in terms of their momentum space Fourier transforms are given by

$$
G_{\phi}\left(x-x^{\prime}\right)=i \int \frac{d^{3} \mathbf{k}}{(2 \pi)^{3}} \mathrm{e}^{i \mathbf{k} \cdot\left(\mathbf{x}-\mathbf{x}^{\prime}\right)}\left(\begin{array}{c}
G_{\phi}^{++}\left(\mathbf{k}, t-t^{\prime}\right) G_{\phi}^{+-}\left(\mathbf{k}, t-t^{\prime}\right) \\
G_{\phi}^{-+}\left(\mathbf{k}, t-t^{\prime}\right) G_{\phi}^{--}\left(\mathbf{k}, t-t^{\prime}\right)
\end{array}\right)
$$

where

$$
\begin{aligned}
& G_{\phi}^{++}\left(\mathbf{k}, t-t^{\prime}\right)=G_{\phi}^{>}\left(\mathbf{k}, t-t^{\prime}\right) \theta\left(t-t^{\prime}\right)+G_{\phi}^{<}\left(\mathbf{k}, t-t^{\prime}\right) \theta\left(t^{\prime}-t\right), \\
& G_{\phi}^{--}\left(\mathbf{k}, t-t^{\prime}\right)=G_{\phi}^{>}\left(\mathbf{k}, t-t^{\prime}\right) \theta\left(t^{\prime}-t\right)+G_{\phi}^{<}\left(\mathbf{k}, t-t^{\prime}\right) \theta\left(t-t^{\prime}\right), \\
& G_{\phi}^{+-}\left(\mathbf{k}, t-t^{\prime}\right)=G_{\phi}^{>}\left(\mathbf{k}, t-t^{\prime}\right), \\
& G_{\phi}^{-+}\left(\mathbf{k}, t-t^{\prime}\right)=G_{\phi}^{<}\left(\mathbf{k}, t-t^{\prime}\right) .
\end{aligned}
$$

The finite-temperature, real-time propagator $G_{\phi}^{++}\left(\mathbf{k}, t-t^{\prime}\right)$ can be written in terms of the spectral function $\rho\left(\mathbf{k}, k_{0}\right)$ [20]

$$
G_{\phi}^{++}\left(\mathbf{k}, t-t^{\prime}\right)=\int_{-\infty}^{+\infty} \frac{d k^{0}}{2 \pi} \mathrm{e}^{-i k^{0}\left(t-t^{\prime}\right)} \rho\left(\mathbf{k}, k^{0}\right)\left\{\left[1+n\left(k^{0}\right)\right] \theta\left(t-t^{\prime}\right)+n\left(k^{0}\right) \theta\left(t^{\prime}-t\right)\right\}
$$

where $n\left(k^{0}\right)$ is the distribution function. 
To account for interactions with the surrounding particles of the thermal bath, particles must be substituted by quasiparticles and dressed propagators are to be adopted (the use of the full corrected propagators should be done with some care to avoid an overcounting of diagrams [21]). Self-energy corrections at one- or two-loops to the propagator modify the dispersion relations and introduce nontrivial effects (damping) due to the imaginary contributions to the self-energy: $\Sigma(k)=\operatorname{Re} \Sigma(k)+i \operatorname{Im} \Sigma(k)$ [21]. Due to the nonvanishing $\operatorname{Im} \Sigma, \rho\left(\mathbf{k}, k^{0}\right)$ acquires in the weak limit a finite width

$$
\begin{aligned}
\Gamma(k) & =-\frac{\operatorname{Im} \Sigma(\mathbf{k}, \omega)}{2 \omega(k)}, \\
\omega^{2}(k) & =\mathbf{k}^{2}+m^{2}+\operatorname{Re} \Sigma(\mathbf{k}, \omega),
\end{aligned}
$$

where $m$ indicates the tree-level mass, and is expressed by

$$
\rho\left(\mathbf{k}, k^{0}\right)=i\left[\frac{1}{\left(k^{0}+i \varepsilon+i \Gamma\right)^{2}-\omega^{2}(k)}-\frac{1}{\left(k^{0}-i \varepsilon-i \Gamma\right)^{2}-\omega^{2}(k)}\right] .
$$

It is easy to show that the free propagator is obtained when taking the limit $\Gamma \rightarrow 0$. Equation (6) has four poles in the complex plane $q^{0}$ plane given by $\omega \pm i \Gamma$ and $-\omega \pm i \Gamma$. Performing the integration over $k^{0}$ in the Eq. (4), one gets [20

$$
\begin{aligned}
G_{\phi}^{>}\left(\mathbf{k}, t-t^{\prime}\right) & =\frac{1}{2 \omega}\left\{[1+n(\omega-i \Gamma)] \mathrm{e}^{-i(\omega-i \Gamma)\left(t-t^{\prime}\right)}+n(\omega+i \Gamma) \mathrm{e}^{-i(\omega+i \Gamma)\left(t-t^{\prime}\right)}\right\}, \\
G_{\phi}^{<}\left(\mathbf{k}, t-t^{\prime}\right) & =G_{\phi}^{>}\left(\mathbf{k}, t^{\prime}-t\right) .
\end{aligned}
$$

In what follows we shall adopt dressed propagators to compute the thermal averages of composite operators, allowing us to naturally and self-consistently include the effects of the incoherent nature of plasma physics and to show that $C P$-violating quantities vanish in the limit of fast interactions: as already evident from expression (7), $\tau \simeq \Gamma^{-1}$ is a natural temporal cut-off and any contribution at times $t_{x}<t_{z}$ to a $C P$-violating observable computed at the instant $t_{z}$ should be expected to vanish for $\left(t_{z}-t_{x}\right) \lesssim \tau$.

We are now in the position to compute $C P$-violating sources resulting from particle interaction with an expanding bubble during a first-order electroweak phase transition. Our starting point is the CPT finite temperature generating functional for the 1PI Green's functions with insertion of a composite operator $\hat{O}(z)$ (in the following $\hat{O}(z)$ will represent a particle current) [1] 


$$
\Gamma\left[\phi_{\mathrm{cl}}^{\alpha}, J_{O}\right]=W\left[J^{\alpha}, J_{O}\right]-\sum_{\beta} \int_{c} d^{4} x J_{\beta}(x) \phi_{\mathrm{cl}}^{\beta}(x)
$$

where the $\phi_{\mathrm{cl}}$ 's are the different classical fields of the theory with sources $J$ 's and will be labelled with greek indices, $J_{O}$ is the source for the operator $\hat{O}$ and the subscript $c$ on the time contour reminds that the closed-time path has been chosen [19]. Note that the Legendre transformation has been performed only on the fields and not on the composite operator [22]. To avoid any confusion, we point out that the terminology classical fields should be understood in the QFT sense [22], i.e. the $\phi_{\mathrm{cl}}$ 's are the fields which extremize the combination

$$
\Gamma\left[\phi^{\alpha}, J_{O}\right]+\sum_{\beta} \int_{c} d^{4} x J_{\beta}(x) \phi^{\beta}(x)
$$

and from now on we will abolish the suffice cl.

According to the time contour, any generic quantity $X$ is doubled to $X_{+}$and $X_{-}$. Although $X_{+}$and $X_{-}$are actually the same, one has to regard them different from each other for technical reasons. The association of $X_{\mathrm{c}}=\left(X_{+}+X_{-}\right) / 2$ with the physical field imposes we take $X_{\Delta}=X_{+}-X_{-}=0$ at the end of the calculation [19.

The quantity we are interested in is the (temporal evolution of the) expectation value $O_{\mathrm{c}}(z)$ of the operator $\hat{O}(z)$ on the background given by the fields $\phi_{\mathrm{c}}^{\alpha}$ :

$$
\begin{aligned}
O_{\mathrm{c}}(z) & =\frac{O_{+}(z)+O_{-}(z)}{2} \\
O_{ \pm}(z) & =\left.\frac{1}{ \pm i} \frac{\delta \Gamma\left[\phi_{+}^{\alpha}, \phi_{-}^{\alpha}, J_{O}^{ \pm}\right]}{\delta J_{O}^{ \pm}(z)}\right|_{J_{O}^{+}=J_{O}^{-}=0}=\mathcal{O}_{ \pm}\left[\phi_{+}^{\alpha}, \phi_{-}^{\alpha}\right](z) .
\end{aligned}
$$

The minus sign in front of the functional derivative $\delta / \delta J_{O}^{-}$takes into account that time now runs only forward.

Following a standard procedure in QFT, we can now expand the (finite temperature) generating functional $\Gamma\left[\phi^{\alpha}, J_{O}\right]$ in terms of the classical fields of the theory $\phi_{ \pm}^{\alpha}(x)$ [22]

$$
\Gamma\left[\phi^{\alpha}, J_{O}\right]=\sum_{n=0}^{\infty} \sum_{i_{1}, \cdots, i_{n}} \frac{1}{n !} \int_{c} d^{4} x_{1} \cdots d^{4} x_{n} \Gamma_{i_{1}, \cdots, i_{n}}^{(n)}\left(x_{1}, \cdots, x_{n}\right) \phi_{i_{1}}\left(x_{1}\right) \cdots \phi_{i_{n}}\left(x_{n}\right),
$$

where, with a shorthand notation, we have indicated through the indices $i_{1}, \cdots i_{n}$ all the possible combinations of the scalar fields of the theory $\phi_{ \pm}^{\alpha}$, and the coefficients of the expansion 


$$
\Gamma_{i_{1}, \cdots, i_{n}}^{(n)}\left(x_{1}, \cdots, x_{n}\right)=\left.\frac{\delta^{(n)} \Gamma\left[\phi_{+}^{\alpha}, \phi_{-}^{\alpha}, J_{O}^{ \pm}\right]}{\delta \phi_{i_{1}}\left(x_{1}\right) \cdots \delta \phi_{i_{n}}\left(x_{n}\right)}\right|_{\phi_{ \pm}^{\alpha}=0}
$$

are the $n$-point 1PI Green's functions computed for vanishing $\phi_{ \pm}^{\alpha}(x)$.

The functional $\mathcal{O}_{ \pm}\left[\phi_{+}^{\alpha}, \phi_{-}^{\alpha}\right](z)$ can then be written in a power series of $\phi_{ \pm}^{\alpha}$

$\mathcal{O}_{ \pm}\left[\phi_{+}^{\alpha}, \phi_{-}^{\alpha}\right](z)=\sum_{n=0}^{\infty} \sum_{i_{1}, \cdots, i_{n}} \frac{1}{n !} \int_{c} d^{4} x_{1} \cdots d^{4} x_{n} \mathcal{O}_{i_{1}, \cdots, i_{n}}^{(n)}\left(x_{1}, \cdots, x_{n} ; z\right) \phi_{i_{1}}\left(x_{1}\right) \cdots \phi_{i_{n}}\left(x_{n}\right)$.

The coefficients of the expansion (13) are the $n$-point 1PI Green's functions with one insertion of the operator $\hat{O}_{ \pm}$computed for vanishing $\phi_{ \pm}^{\alpha}(x)$

$$
\mathcal{O}_{i_{1}, \cdots, i_{n}}^{(n)}\left(x_{1}, \cdots, x_{n} ; z\right)=\left.\frac{1}{ \pm i} \frac{\delta^{(n+1)} \Gamma\left[\phi_{+}^{\alpha}, \phi_{-}^{\alpha}, J_{O}^{ \pm}\right]}{\delta \phi_{i_{1}}\left(x_{1}\right) \cdots \delta \phi_{i_{n}}\left(x_{n}\right) \delta J_{O}^{ \pm}(z)}\right|_{\phi_{ \pm}^{\alpha}=J_{O}^{+}=J_{O}^{-}=0}
$$

and can be used to compute through a diagrammatic approach the temporal evolution of the vacuum expectation value $O_{\mathrm{c}}(z)$ of the operator $\hat{O}(z)$ in a generic space-time dependent background described by the classical fields $\phi_{ \pm}^{\alpha}(x)$. For our practical purposes, such a background will be the bubble wall configuration expanding in the thermal bath with velocity $v_{\mathrm{w}}$, which extremizes the combination (9) with vanishing sources.

Thus, given a certain theory with the necessary amount of $C P$-violation and predicting nonequilibrium conditions at a certain space-time point through the passage of the bubble wall, the general formulae (10-14) can be applied to compute in the rest frame of the thermal bath and at a certain point $\mathbf{z}$ the temporal evolution of the nonequilibrium expectation values $J_{\mathbf{c}}^{\mu}\left(t_{z}, \mathbf{z}\right)$ of the current operators $J^{\mu}\left(t_{z}, \mathbf{z}\right)$ for the different particles of the theory.

Even if the general formalism described so far might seem rather cumbersome, we shall see through a practical example that it leads to the computation of physical quantities whose meaning is clear and well-established, making the physical interpretation of the whole picture rather intuitive. Moreover, the expansion (13) is adequate to demonstrate the physics of quantum interference required to generate $C P$-violating observables. It originates a series of powers in the classical Higgs fields whose imaginary parts leads to nonvanishing $C P$-violating observables. $C P$-violating sources $\gamma_{Q}(z)$ (per unit volume 
and unit time) of a generic charge $Q$ associated to the current $J^{\mu}(z)$ and accumulated by the moving wall at a point $\mathbf{z}$ of the plasma can then be constructed from $J_{\mathrm{c}}^{\mu}(z)$ [15, [16]

$$
\gamma_{Q}(z)=\partial_{\mu} J_{\mathrm{c}}^{\mu}(z)
$$

We point out that this definition of the $C P$-violating source $\gamma_{Q}(z)$, though adequate to describe the damping effects originated by the plasma interactions, does not involve any relaxation time scale arising when diffusion and particle changing interactions are included. In this paper we will focus on the computation of $C P$-violating observables through a nonequilibrium QFT approach and take the same point of view as in refs. [15,16]: one can leave aside diffusion and particle changing interactions and account for them independently in the rate equations. It is clear, however, that a full nonequilbrium QFT approach to electroweak baryogenesis, based on a complete set of quantum Boltzmann equations, should describe consistently all the effects. Indeed, the CPT formalism, in combination with the so-called non-local source theory and loop expansion techniques developed by Cornwall, Jackiw and Tamboulis [23], could provide the Ginzburg-Landau equation [24] for the order parameter (in our case the Higgs background field) and the generalized Dyson-Schwinger equations which incorporate the initial state correlations and provide a systematic treatment of the quantum correlations to any order of perturbation theory. This procedure would yield two distinct equations for each of the Wigner functions: the renormalization equation and the transport equation. The latter should account in a self-consistent way for all the effects (diffusion, $C P$-violation, damping, particle changing interactions, etc.) giving rise to the final baryon asymmetry. This analysis is, however, beyond the scope of the present work.

Due to their generality, expressions (10-14) are valid for a generic wall shape and size $L_{\mathrm{w}}$ and are not based on any assumptions on the relative magnitude of the mean free paths and thickness of the bubble wall. In this respect, the method described in this paper should be regarded as an extension of the linear response method [25] (as accurate as the thick wall approximation is made) to compute the charge current densities produced from an initial $C P$-symmetric thermal particle distribution when space-time dependent $C P$ violating terms are turned on in the Hamiltonian for a time equal to the thermalization time. 


\section{An example of construction of a $C P$-violating source}

Let us now illustrate the method by means of a specific situation: the two-Higgs doublet model; more in particular, we will work out the $C P$-violating source constructed from the Higgs current operator $J_{1}^{\mu}(z)$ associated to the neutral Higgs field $H_{1}^{0}$

$$
J_{1}^{\mu}(z)=i\left(H_{1}^{\dagger} \partial^{\mu} H_{1}-\partial^{\mu} H_{1}^{\dagger} H_{1}\right)
$$

The reader should keep in mind that the method applies to other theories as well.

The most general tree level scalar potential for the two-Higgs doublet model is given by

$$
\begin{aligned}
V & =m_{1}^{2}\left|H_{1}\right|^{2}+m_{2}^{2}\left|H_{2}\right|^{2}-\left(m_{3}^{2} H_{1} H_{2}+\text { h.c. }\right)+\lambda_{1}\left|H_{1}\right|^{4}+\lambda_{2}\left|H_{2}\right|^{4} \\
& +\lambda_{3}\left|H_{1}\right|^{2}\left|H_{2}\right|^{2}+\lambda_{4}\left|H_{1} H_{2}\right|^{2}+\left[\lambda_{5}\left(H_{1} H_{2}\right)^{2}+\lambda_{6}\left|H_{1}\right|^{2} H_{1} H_{2}+\lambda_{7}\left|H_{2}\right|^{2} H_{1} H_{2}+\text { h.c. }\right] .
\end{aligned}
$$

Of the two phases of the classical vacuum expectation values of the Higgs fields $\delta_{1}$ and $\delta_{2}$, defined by $H_{\mathrm{c}, i}(x)=v_{i}(x) \exp \left[i \delta_{i}(x)\right](i=1,2)$, only the gauge invariant combination $\theta=\delta_{1}+\delta_{2}$ appears in the scalar potential, whereas the orthogonal combination represents the gauge phase. We assume that the parameters of the Lagrangian are such that, when loop corrections are considered, the potential assumes a double-well shape and expanding bubble wall solution exists described by the configuration $H_{\mathrm{c}, i}(x)$.

The interesting dynamics for baryogenesis takes place in a region close to or inside the bubble wall and we approximate it with an infinite plane traveling at a constant speed $v_{\mathrm{w}}$ along the $z$-axis.

Since the coefficients of the expansion (13) must be computed for vanishing $\phi_{ \pm}^{\alpha}(x)$, we must deal with resummation of the propagators of the Higgs fields in order to deal with infrared divergencies [26]. In the unbroken phase, the Higgs spectrum contains two complex electrically neutral fields and two charged ones. At the tree-level, the squared masses of one of the neutral states and one of the charged ones are negative, since the origin of the field space becomes a minimum of the effective potential only after inclusion of the finite temperature corrections. The resummation can be achieved by considering the propagators for the eigenstates of the thermal mass matrix, which has positive eigenvalues given by 


$$
M_{h, H}^{2}(T)=\frac{m_{1}^{2}(T)+m_{2}^{2}(T) \mp \sqrt{\left(m_{1}^{2}(T)-m_{2}^{2}(T)\right)^{2}+4 m_{3}^{4}(T)}}{2},
$$

where the $m_{i}^{2}(T)$ are the thermal corrected mass parameters of the potential (17), $m_{1}^{2}(T) \simeq m_{1}^{2}+(3 / 16) g^{2} T^{2}, m_{2}^{2}(T) \simeq m_{2}^{2}+(1 / 4) h_{t}^{2} T^{2}$, while $m_{3}^{2}(T)$ receives only logarithmic corrections in $T$ [7]. Here $g$ and $h_{t}$ are the $S U(2)_{L}$ gauge coupling and the top Yukawa coupling, respectively. Correspondingly, the neutral complex eigenstates are given by

$$
\left\{\begin{array}{l}
h=\cos \beta_{h H} H_{1}^{0}+\sin \beta_{h H} H_{2}^{0 *}, \\
H=-\sin \beta_{h H} H_{1}^{0}+\cos \beta_{h H} H_{2}^{0 *},
\end{array}\right.
$$

where

$$
\sin 2 \beta_{h H}=\frac{2 m_{3}^{2}(T)}{M_{h}^{2}(T)-M_{H}^{2}(T)} .
$$

Completely analogous formulae hold for the charged eigenstates.

Given the classical action (remind that time runs only forward)

$$
S\left[H_{1}, H_{2}\right]=\int d^{4} x\left\{\mathcal{L}\left[H_{1,+}, H_{2,+}\right]-\mathcal{L}\left[H_{1,-}, H_{2,-}\right]\right\},
$$

it is not difficult to show that the the vacuum expectation value $J_{1, \mathrm{c}}^{\mu}(z)$ gets (beyond the tree level ones) contributions from four different one-loop Feynman diagrams, symbolically depicted in Fig. 1, which are obtained by assigning in all the possible ways the space-time points $x$ and $z$ on the positive or negative time branches. One gets

$$
J_{1, \mathrm{c}}^{\mu}(z)=\frac{J_{1,+}^{\mu}(z)+J_{1,-}^{\mu}(z)}{2}=-4 \lambda_{5} \sin 2 \beta_{h H} \int d^{4} x H(x) \Sigma^{\mu}(x, z)+\mathcal{O}\left[\left(H_{\mathrm{c}} / T\right)^{4}\right],
$$

where we have made an expansion in the parameter $\left(H_{\mathrm{c}} / T\right)$. The function

$$
\Sigma^{\mu}(x, z)=\theta\left(t_{z}-t_{x}\right) \lim _{y \rightarrow z}\left\{\partial_{y}^{\mu} \operatorname{Im}\left[G_{H}^{++}(y-x) G_{h}^{++}(x-z)\right]\right\}-(h \leftrightarrow H)
$$

is expressed in terms of the retarded self-energy Green's function and we have defined

$$
H(x)=\operatorname{Im}\left(H_{1, \mathrm{c}}^{0} H_{2, \mathrm{c}}^{0}\right)(x) .
$$

Notice that, being dependent on $H(x), J_{1, \mathrm{c}}^{\mu}$ is vanishing if no $C P$-violation is present in the scalar sector. 
Using the spectral representation (6), we can cast the function $\Sigma^{\mu}(x, z)$ into the form

$$
\begin{aligned}
\Sigma^{\mu}(x, z) & =-i \theta\left(t_{z}-t_{x}\right) \int \frac{d^{4} q_{1}}{(2 \pi)^{4}} \int \frac{d^{4} q_{2}}{(2 \pi)^{4}} q_{1}^{\mu} \rho_{H}\left(q_{1}\right) \rho_{h}\left(q_{2}\right) \\
& \times\left[n_{h}\left(q_{2}^{0}\right)-n_{H}\left(q_{1}^{0}\right)\right] \mathrm{e}^{-i(z-x) \cdot\left(q_{1}-q_{2}\right)}-(h \leftrightarrow H) .
\end{aligned}
$$

Performing the $q_{1}^{0}$ and $q_{2}^{0}$ integrations, one can easily show that $J_{1, \mathrm{c}}^{\mu}(z)$ is vanishing in the limit of constant space-time $C P$-violating background $H(x)$ : this is the very wellknown result that $C P$-violating sources arise only in a nontrivially space-time dependent $C P$-violating Higgs background.

The presence of the retarded Green's function carrying the $\theta\left(t_{z}-t_{x}\right)$ makes the physical interpretation of expression (22) rather intuitive: let us imagine the bubble wall configuration moving along the $z$-axis and divided into many strips along the same axis; when each of these strips passes from $t_{x}$ to $t_{x}+d t_{x}$ through a small volume element centered at the point $\mathbf{z}$ in the thermal bath, it deposits there a certain amount of charge and the vacuum expectation value of $J_{1}^{\mu}$ at the time $t_{z}$ turns out to be the sum of all these contributions received for times $t_{x}<t_{z}$. Moreover, because of the presence of a finite damping rate $\tau^{-1}$, the strips' contributions are not all equivalent, but they will be weighted by the exponential factors present in the Green's functions, thus making the strips'contributions negligible for times $\left(t_{z}-t_{x}\right) \gtrsim \Gamma^{-1}$.

The advantage of the method illustrated in this paper is that very general formulae, like Eqs. (22-23), can be obtained for the temporal evolution of $C P$-violating observables without any particular assumption on the relative magnitude of the mean free paths and the thickness of the wall, and on the particle distribution functions (eventually determined by the Boltzmann equations of motion). The standard thin wall and thick wall limits are recovered in the limit $\Gamma L_{\mathrm{w}} \rightarrow 0$ and $\infty$, respectively, once the Green's functions (7) are inserted in the expression (23) for $\Sigma^{\mu}(x, z)$.

In order to deal with analytic expressions, we can work out the thick wall limit and simplify expressions (22-24) by performing a derivative expansion

$$
H(x) \simeq H(z)+\left.\partial_{x}^{\nu} H\right|_{x=z}\left(x_{\nu}-z_{\nu}\right)+\mathcal{O}\left[\left(\Gamma_{h, H} L_{\mathrm{w}}\right)^{2}\right] .
$$

This expansion is valid only when the mean free paths $\tau \simeq \Gamma^{-1}$ are smaller than the scale of variation of the Higgs background determined by the wall thickness $L_{\mathrm{w}}$. An 
estimate of the Higgs damping rate in the Standard Model was obtained in ref. [27] in the low momentum limit and can be used here only to give very crude estimate of the Higgs coherent times, $\tau^{-1} \sim\left(10^{-2}-10^{-1}\right) T$ (with such values, our derivative expansion is not perfectly justified since the wall thickness can span the range $(10-100) / T$. Its benefit relies on the possibility to obtain analytical expressions in $\Gamma$ and we adopt it for pedagogical purposes).

To work out exactly the vacuum expectation value of the Higgs current $J_{1, \mathrm{c}}^{\mu}(z)$ one should know the exact form of the distribution functions which, in ultimate analysis, is provided by solving the Boltzmann equations. However, any departure from thermal equilibrium distribution functions is caused at a given point by the passage of the wall and, therefore, is $\mathcal{O}\left(v_{\mathrm{w}}\right)$. Working with thermal equilibrium distribution functions in Eq. (7) amounts to ignoring terms of order $v_{\mathrm{w}}^{2} \lesssim 1$, which is as accurate as the bubble wall is moving slowly in the plasma and we shall adopt this approximation from now on. At this order, the $G_{\phi}^{++}\left(\mathbf{k}, t-t^{\prime}\right)$ Green's function becomes

$$
\begin{aligned}
G_{\phi}^{++}\left(\mathbf{k}, t-t^{\prime}\right) & =\frac{\mathrm{e}^{-\Gamma\left|t-t^{\prime}\right|}}{2 \omega[\cosh (\beta \omega)-\cos (\beta \Gamma)]}\left\{\sinh (\beta \omega) \cos \left(\omega\left|t-t^{\prime}\right|\right)\right. \\
& \left.+\sin (\beta \Gamma) \sin \left(\omega\left|t-t^{\prime}\right|\right)+i[\cos (\beta \Gamma)-\cosh (\beta \Gamma)] \sin \left(\omega\left|t-t^{\prime}\right|\right)\right\} .
\end{aligned}
$$

To demonstrate the conflict between the incoherent nature of fast plasma interactions and the coherent phenomenon of $C P$-violation, we can take the ideal limit $\Gamma_{h} \simeq \Gamma_{H} \gg T$. Even if the physical lower limit in the temporal integration is about $t_{z}-L_{\mathrm{w}}$, we can safely take it $-\infty$ in the thick wall approximation. Using expressions (22-27) one can show that

$$
\begin{aligned}
\gamma_{1}(z) & =\partial_{\mu} J_{1, \mathrm{c}}^{\mu}(z) \simeq-2 \lambda_{5} m_{3}^{2} \partial_{t_{z}} H(z) \int_{-\infty}^{t_{z}} d t_{x}\left(t_{z}-t_{x}\right) \Gamma_{h}^{2} \mathrm{e}^{-2 \Gamma_{h}\left(t_{z}-t_{x}\right)} \int \frac{d^{3} \mathbf{k}}{(2 \pi)^{3}} \frac{\sin \left(\beta \Gamma_{h}\right)}{\omega_{h}^{4}} \\
& \times\left[\cos \left(\omega_{h}+\omega_{H}\right)\left(t_{z}-t_{x}\right)-\cos \left(\omega_{h}-\omega_{H}\right)\left(t_{z}-t_{x}\right)\right]+\mathcal{O}\left[v_{\mathrm{w}}^{2},\left(\Gamma_{h} L_{\mathrm{w}}\right)^{2},\left(M_{h, H} / T\right)^{4}\right] .
\end{aligned}
$$

Performing the integration in $t_{x}$ one gets

$$
\gamma_{1}(z) \simeq \lambda_{5} \frac{\sin \left(\beta \Gamma_{h}\right)}{2 \pi^{2}}\left(\frac{T m_{3}^{2}}{\Gamma_{h}^{2}}\right) \partial_{t_{z}} H(z) \mathcal{I}_{h}\left(T, \Gamma_{h}\right)+\mathcal{O}\left[v_{\mathrm{w}}^{2},\left(\Gamma_{h} L_{\mathrm{w}}\right)^{2},\left(M_{h, H} / T\right)^{4}\right]
$$

where

$$
\mathcal{I}_{h}\left(T, \Gamma_{h}\right)=\int_{0}^{\infty} d x \frac{x^{2}}{\left(x^{2}+y^{2}\right)} \frac{1}{\cosh \left(\sqrt{x^{2}+y^{2}}\right)-\cos \left(\beta \Gamma_{h}\right)}
$$




$$
\begin{aligned}
& \simeq \frac{2}{\sin \left(\beta \Gamma_{h}\right)} \operatorname{arctg}\left(\frac{\sin \beta \Gamma_{h}}{1-\cos \beta \Gamma_{h}}\right)+\mathcal{O}\left(y^{2}\right), \\
y & =M_{h}(T) / T .
\end{aligned}
$$

The steep dependence of $\gamma_{1}(z)$ on $\Gamma_{h}$ reflects the high suppression coming from decoherence due to incoherent scatterings in the plasma whose rate increases with $\Gamma_{h}$. The origin of this strong suppression is similar to the one forbidding electroweak baryogenesis in SM [5] and is evident from Eq. (28): due to the exponential cut-off exp $\left[-\Gamma_{h} t\right]$, as the wall crosses a small volume element of the plasma the current density deposited at the instant $t_{z}$ into it may receive relevant contributions only from those sheets of the bubble wall which pass through the volume element at times in the range $\left[t_{z}-\tau_{h}, t_{z}\right]$, which vanishes for very small interaction times $\tau_{h}$.

In the opposite limit of very weak interactions $\Gamma_{h} \lesssim T$, a straightforward calculation leads to

$$
\gamma_{1}(z) \simeq \frac{\lambda_{5}}{32 \pi} \Gamma_{h} T \frac{m_{3}^{2}}{M_{h}^{3}(T)} \partial_{t_{z}} H(z)+\mathcal{O}\left[v_{\mathrm{w}}^{2},\left(\Gamma_{h} L_{\mathrm{w}}\right)^{2},\left(M_{h, H} / T\right)^{4}\right] .
$$

Eq. (31) warrants some comments. First, we notice that the momentum integration giving rise to the $1 / M_{h}^{3}$ dependence in the above expression is infrared dominated: quasiparticles with long wavelengths and incident perpendicularly to the wall give a large contribution to $\gamma_{1}(z)$ and a classical approximation is not adequate to describe the quantum interference nature of $C P$-violation. Secondly, the vanishing of $\gamma_{1}(z)$ for very small interaction rates $\Gamma_{h} \ll T$ is in agreement with what found in ref. [15] and should be expected since the propagation is semi-classical in that limit (even if one should not expect to recover our result by a complete classical treatment, because the momentum integral is dominated by particles with long wavelengths for which a full classical approach is inappropriate). Working in the thick wall limit and with very small damping rates makes (contrary to what happens for strong damping rates) all the different sheets of the bubble wall equivalent. In these limits, there are no time-scales involved and the amount of charge deposited in a small volume element turns out to be approximately independent of time, resulting in a vanishing $\gamma_{1}(z)$. As a matter of fact, such a behaviour for very small damping rates should not be completely trusted since our derivative expansion may be applied only for $\Gamma_{h} \gtrsim L_{\mathrm{w}}^{-1}$. Also, as we mentioned above, our computation tacitly 
assumes large relaxation times provided by the diffusion properties of the particles in the plasma. When considering the effects of finite relaxation times, the behaviour of $\gamma_{1}(z)$ for $\Gamma_{h} \ll T$ should differ from the one obtained here since particles are allowed to diffuse away from the region centered at the point $\mathbf{z}$.

\section{Conclusions}

In this paper we have proposed a general method to compute $C P$-violating observables in the context of electroweak baryogenesis. It is valid for all shapes of the expanding bubble wall and relies on a nonequilibrium quantum field theory diagrammatic approach. Dressed propagators have been adopted to account for the interactions with the surrounding particles, which modify the dispersion relation and introduce damping effects. The suppression of $C P$-violating sources resulting from fast incoherent thermal processes has been recovered in a direct way. The method is complementary to the one introduced in refs. [15,16] and reproduce qualitatively their results. Our computation based on nonequilibrium QFT should be regarded as a first step towards a complete understanding of the dynamics of the electroweak baryogenesis by means of a complete nonequilibrium quantum kinetic theory approach. Indeed, only the latter should be able to properly describe the kinematics of quasiparticle excitations in the infrared energy region, i.e. excitations whose Compton wavelength is larger both than the typical interaction time and the bubble wall width. For such quasipartilces, the classical approximation to the Boltzmann equation is not adequate. We hope to address this problem in the next future.

\section{ACKNOWLEDGMENTS}

It is a pleasure to thank A.E. Nelson for reading the manuscript and very useful discussions. M. Pietroni is also acknowledged for criticisms. 


\section{REFERENCES}

[1] D. Boyanovsky, H.J. de Vega, Phys. Rev. D47 (1993) 2343; D. Boyanovsky, D.-S. Lee and A. Singh, Phys. Rev. D48 (1993) 800; D. Boyanovsky, H.J. de Vega and R. Holman, Phys. Rev. D49 (1994) 2769 and Proceedings of the Second Paris Cosmology Colloquium, Observatoire de Paris, June 1994, p. 125-215, H.J. de

Vega and N. Sanchez Editors, World Scientific, 1995; L. Kofman, A.D. Linde and A.A. Starobinsky, Phys. Rev. Lett. 73 (1994) 3195; Y. Shtanov, J. Traschen and R. Brandenberger, Phys. Rev. D51 (1995) 5438; A. Dolgov and K. Freese, Phys. Rev. D51 (1995) 2693; D. Boyanovsky, H.J. de Vega, R. Holman, D.-S. Lee and A. Singh, Phys. Rev. D51 (1995) 4419; D. Boyanovsky, M. D’Attanasio, H.J. de Vega, R. Holman and D.-S. Lee and A. Singh, hep-ph/9505220; D. Boyanovsky, M. D'Attanasio, H.J. de Vega, R. Holman and D.-S. Lee, hep-ph/9507414.

[2] A.G. Cohen, D.B. Kaplan, and A.E. Nelson, Annu. Rev. Nucl. Part. Sci. 43 (1993) 27; A. Dolgov, Phys. Rep. 222, (1992) 311.

[3] For a review see, M. Quirós, Field theories at finite temperature and Phase Transitions, Helvetica Physica Acta 67 (1994) 451 and references therein.

[4] G.R. Farrar and M.E. Shaposhnikov, Phys. Rev. Lett. 70 (1993) 2833 and Phys. Rev. D50 (1994) 774.

[5] M.B. Gavela et al., Mod. Phys. Lett. A9 (1994) 795 and Nucl. Phys. B430 (1994) 382; P. Huet and H. Sather, Phys. Rev. D51 (1995) 379.

[6] A.G. Cohen, D.B. Kaplan, and A.E. Nelson, Phys. Lett. B263 (1991) 86.

[7] D. Comelli and M. Pietroni, Phys. Lett. B306 (1993) 67; D. Comelli and M. Pietroni and A. Riotto, Nucl. Phys. B412 (1994) 441; Phys. Rev. D50 (1994) 7703; Phys. Lett. 343 (1995) 207; J.R. Espinosa, J.M. Moreno, M. Quirós, Phys. Lett. B319 (1993) 505.

[8] A.G. Cohen, D.B. Kaplan, and A.E. Nelson, Nucl. Phys. B373 (1992) 453; Phys. Lett. 294 (1992) 57.

[9] V.A. Kuzmin, V.A. Rubakov and M.E. Shaposhnikov, Phys. Lett. B155 (1985) 36. 
[10] L. Mc Lerran et al., Phys. Lett. B256 (1991) 451; M. Dine, P. Huet and R. Singleton Jr., Nucl. Phys. B375 (1992) 625; M. Dine et al., Phys. Lett. B257 (1991) 351; A.G. Cohen and A.E. Nelson, Phys. Lett. B297 (1992) 111.

[11] D. Comelli, M. Pietroni and A. Riotto, Phys. Lett. B354 (1995) 91 and DESY 95-109 preprint, submitted to Phys. Rev. D.

[12] S.Yu. Khlebnikov, Phys. Lett. B300 (1993) 376; A.G. Cohen, D.B. Kaplan, and A.E. Nelson, Phys. Lett. B294 (1992) 57; J.M. Cline and K. Kainulainen, Phys. Lett. B356 (1995) 19.

[13] A.G. Cohen, D.B. Kaplan, and A.E. Nelson, Phys. Lett. 336 (1994) 41.

[14] M. Joyce, T. Prokopec and N. Turok, hep-ph 9401351 and hep-ph 9408339; D. Comelli, M. Pietroni and A. Riotto, hep-ph 9406369, to appear in Astroparticle Phys. 4 (1995).

[15] P. Huet and A.E. Nelson, Phys. Lett. B355 (1995) 229.

[16] P. Huet and A.E. Nelson, UW/PT 95-07 preprint, hep-ph 9506477.

[17] S. Jeon, UW/PT 94-09 preprint, hep-ph 9409250.

[18] E.P. Wigner, Phys. Rev. D40 (1932) 749.

[19] See, for instance, K. Chou, Z. Su, B. Hao and L. Yu, Phys. Rep. 118 (1985) 1 and refs. therein.

[20] N.P. Landsmann and Ch.G. van Weert, Phys. Rep. 145 (1987) 141.

[21] See, for instance, R.R. Parwani, Phys. Rev. D45 (1992) 4695 and refs. therein.

[22] See, for instance, S. Pokorski, Gauge Field Theories Cambridge Univ. Press (1987).

[23] J.M. Cornwall, R. Jackiw and E. Tamboulis, Phys. Rev. D10 (1974) 2428.

[24] V.L. Ginzburg and L.D. Landau, Zh. Eksp. Teor. Fiz. 20 (1950) 1064.

[25] E.M. Lifschitz and L.P. Pitaevkii, Statistical Physics, part 2, Pergamon Press, Oxford (1980); A.L. Fetter and J.D. Walecka, Quantum Theory of Many particle Systems, Mc Graw Hill, New York (1971). 
[26] P. Arnold and O. Espinosa, Phys. Rev. D47 (1993) 3546.

[27] P. Elmfors, K. Enqvist and I. Vilja, Nucl. Phys. 412 (1994) 459. 


\section{Figure Captions}

Fig. 1: The 1-loop contribution to the vacuum expectation value of the Higgs current $J_{1}^{\mu}(z)$ 